\title{
Orbital high resolution magnetic resonance imaging with fast spin echo in the acute stage of Leber's hereditary optic neuropathy
}

\author{
Yukihiko Mashima, Kazuhiro Oshitari, Yutaka Imamura, Suketaka Momoshima, \\ Hayao Shiga, Yoshihisa Oguchi
}

\begin{abstract}
Some evidence suggests that the primary locus of the lesion in Leber's hereditary optic neuropathy (LHON) may be intraocular rather than retrobulbar. To clarify this issue, the condition of the retrobulbar portion of the optic nerve was evaluated in patients with the acute stage of LHON. High resolution MRI with fast spin echo sequences of the optic nerve complex in the orbit was carried out. Five patients with acute stage LHON were compared with seven patients with acute stage optic neuritis. On T2 weighted fast spin echo MRI, signal changes did not appear in the retrobulbar optic nerve complex in acute stage LHON. By comparison, patients with optic neuritis showed pronounced high signals in the optic nerve. Subsequent orbital MRI in the atrophic stages of the same patients with LHON showed an increase in signal intensity in the optic nerve toward the orbital apex in both eyes. The present results support the hypothesis that a primary lesion in LHON may be intraocular.

(F Neurol Neurosurg Psychiatry 1998;64:124-127)
\end{abstract}

Keywords: Leber's hereditary optic neuropathy; MRI; fast spin echo sequence; optic neuritis

Leber's hereditary optic neuropathy (LHON) is a maternally inherited optic neuropathy that is characterised by the acute or subacute loss of central vision. The condition occurs mainly in young males and leads to severe optic atrophy. The site and nature of the acute lesion in LHON have not yet been determined. The name implies that LHON is primarily an optic neuropathy. However, some evidence suggests that the primary locus of the lesion might be intraocular rather than retrobulbar. ${ }^{12}$ Brain MRI provides a means of detecting acute lesions of the optic nerve. It has proved to be a sensitive means of detecting demyelinating optic neuritis, which in the acute stage produces high intensity signals in the retrobulbar segment. ${ }^{3-6}$

Two MRI investigations of the optic nerve of patients with LHON used relatively weak (0.5Tesla) magnets. ${ }^{78}$ In a series of eight cases, the affected nerves in patients in the chronic stage were abnormal, with short inversion time inversion recovery (STIR) sequence. ${ }^{7}$ One other series reported minor or no abnormalities in the optic nerve on T1 weighted MRI in 15 cases in differing stages of the disease. ${ }^{8}$ The MRI findings for the optic nerve in the acute stage of LHON have not been determined. The present report describes high resolution MRI findings in five patients studied in the acute stage of LHON by fast spin echo (FSE) sequence. We compared these findings with those in the atrophic stage of LHON and in acute optic neuritis.

\section{Patients and methods}

We investigated five male patients with LHON who were diagnosed by molecular analysis at Keio University Hospital between 1993 and 1995. Their age at onset ranged from 21 to 38 (mean, 25.8) years. All of them carried the 11778 mutation. In four patients, loss of vision in the second eye occurred within three weeks after the loss of vision in the first eye. In the fifth patient, loss of vision in the right eye was followed in 10 months by involvement of the left eye. Initial MRI was within four weeks of onset of loss of vision in nine eyes of the five patients. At the time of MRI, visual loss was moderate or severe in all patients, with visual acuities between 0.08 and 0.4 and cecocentral scotomas.

Four patients were rescanned five to 11 months after the initial scan. They showed total or partial optic disc pallor in both eyes. Visual loss had progressed with visual acuity falling below 0.1 and the presence of larger, denser cecocentral scotomas in both eyes.

For comparison, seven patients with unilateral optic neuritis, including two with papillitis, were scanned by MRI within three weeks after the onset of visual loss. The mean age of the patients (four males and three females) was 26.4 (range 10-35) years. All showed central scotomas and decreased visual acuities from 


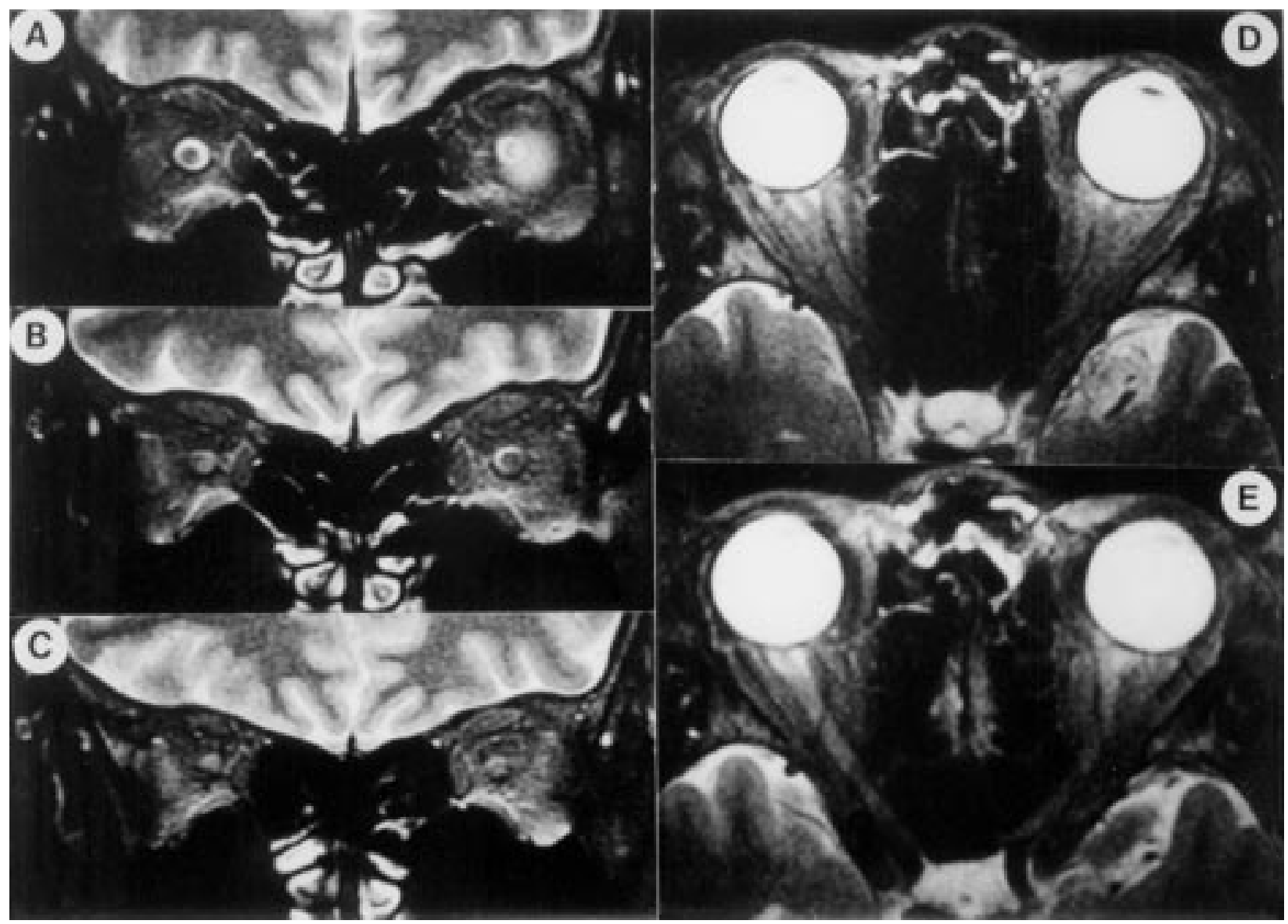

Figure 1 Fast spin echo MRI (4000/104 (repetition time ms/echo time ms)) obtained at 4 mm intervals from the globe in the early acute stage of LHON in both eyes of a 29 year old man. $(A, B)$ Coronal images show a ring of high signal intensity surrounding the optic nerve. (C) Coronal image of the optic nerve complex is obscure. (D) The subarachnoid space is wider behind the globe and narrowed toward the orbital apex on the axial image. The image of the optic nerve complex is obscure. (E) Six months after onset. The optic nerve complex is slightly hyperintense throughout the orbit on the axial image.

hand motion to 0.2 in the affected eye. Visual evoked potentials (VEPs) elicited by pattern stimulus were unrecordable in all seven patients, but the VEPs elicited by a flashing light showed a delayed response in six of the seven patients. They were all treated with steroids and their vision recovered to 1.0 or 1.2 .

Imaging of the orbit with a T2 weighted FSE sequence with frequency selective fat suppression was performed using a 1.5 Tesla magnet (Signa, General Electric Medical Systems, Milwaukee, WI, USA). A five inch circular coil was used for generic surface imaging. The images, $3 \mathrm{~mm}$ slices with a $1 \mathrm{~mm}$ gap (256×192 matrix, $14 \mathrm{~cm}$ field of view) were obtained at a repetition time of $4000 \mathrm{~ms}$ and an echo time of $104 \mathrm{~ms}$, using two excitations. Ten slices in the coronal or axial plane were obtained routinely in two minutes and 12 seconds, which is well under the limit of patients' tolerance. The in plane resolution was as high as $0.73 \mathrm{~mm} \times 0.54 \mathrm{~mm}$ with a $3 \mathrm{~mm}$ slice depth. Gadolinium-diethylenetriaminepentaacetic acid (Gd-DTPA) enhanced T1 weighted MRIs with fat suppression were also obtained in four of the five patients with LHON and five of the seven patients with optic neuritis.

To investigate the anatomical relation between the optic nerve and its sheath, we determined the vertical cross sectional diameter of the optic nerve (L1) and that of the subarachnoid space surrounding the optic nerve (L2) on the first or second coronal MRI slice posterior to the globe. The ratio of L1 to L2 (R) was determined to evaluate the anatomical status of the optic nerve complex, as previously reported. $^{9}$

\section{Results}

MRI of the optic nerve complex in the orbit appeared normal in the acute stage, within four weeks after onset of LHON. The mean $\mathrm{R}$ in this stage was 0.62 (SD 0.04) ( $n=9$ eyes). We previously reported that the mean $\mathrm{R}$ in 15 normal subjects aged 25 to 45 years was 0.61 (SD 0.05). ${ }^{9}$ These values in the patients with LHON did not differ significantly from those of normal subjects (Mann-Whitney $U$ test, $p>0.05)$. No enhancement of Gd-DTPA was found throughout the optic nerve in the orbit of any of the four patients studied. Figure 1 shows the orbital MRI of a 29 year old man in the acute stage of LHON. A ring shaped area of high signal intensity which represents the subarachnoid space with CSF and a central hypointense core of the optic nerve which corresponds to myelinated nerve fibres were seen on two coronal slices posterior to the globe. The image of the optic nerve complex in the middle to posterior portion of the orbit was obscure. The optic nerve signal in this patient 


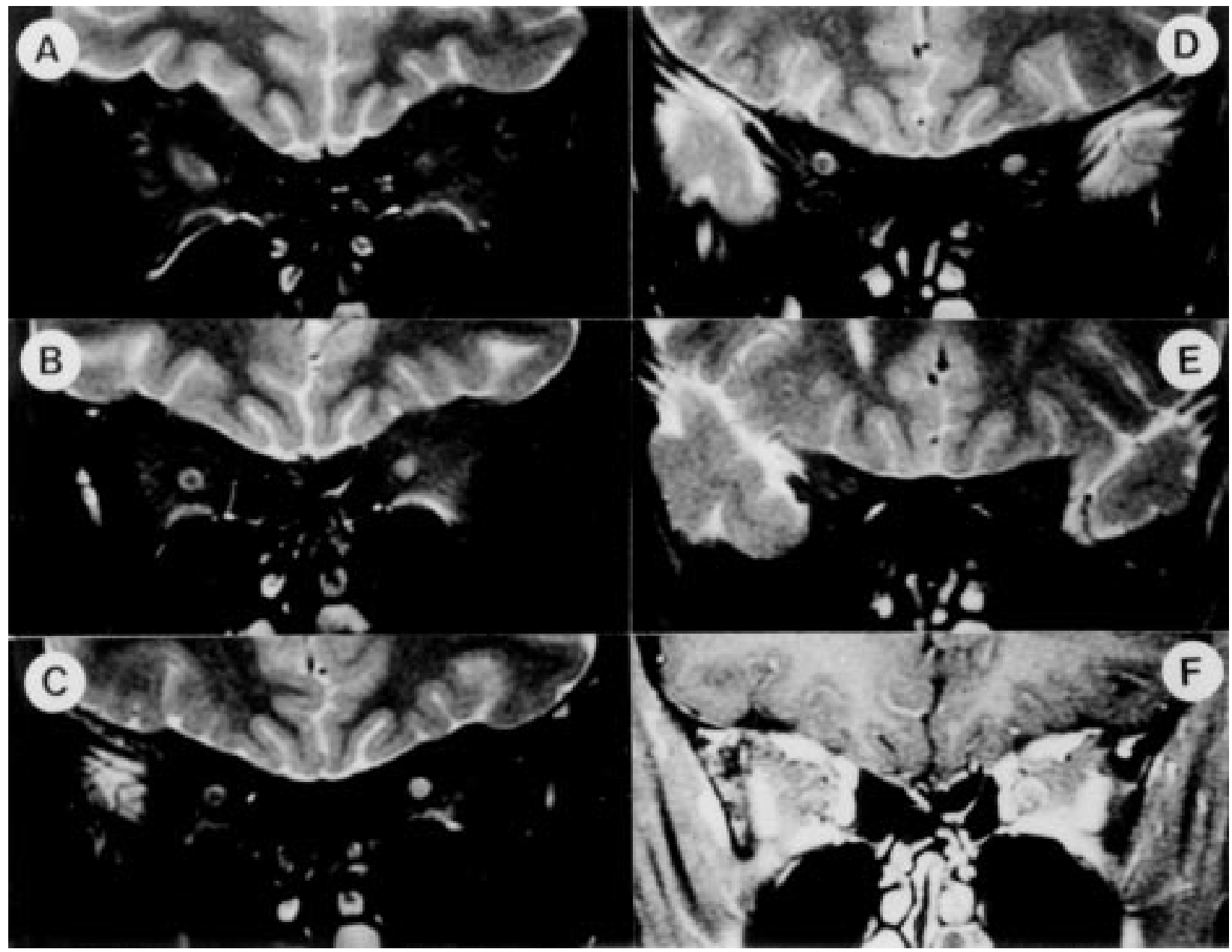

Figure 2 Coronal fast spin echo MRI (4,000/104 (repetition time ms/echo time ms)) obtained at 4 mm intervals from the globe in the early acute stage of optic neuritis in the left eye of a 23 year old woman. (A) Normal appearance of the left optic nerve complex $4 \mathrm{~mm}$ posterior to the globe. (B, $C$, $D$ )

Increased signal intensities in the left eye at 8 to $16 \mathrm{~mm}$ posterior to the globe. (E) Normal appearance of the left optic nerve complex $20 \mathrm{~mm}$ posterior to the globe. (F) Gadolinium-DTPA enhanced T1weighted MRI (380/12) with fat suppression enhances the left optic nerve complex on the same slice as B. The right eye appears normal.

seemed to be normal. One patient who had LHON of differing clinical stages in each eye showed a high intensity signal throughout the orbit in the atrophic eye but a normal signal in the eye in the acute stage of LHON.

Orbital MRI was repeated in the atrophic stages in four patients with LHON. The scans showed an increase in signal intensity in the optic nerve complex toward the orbital apex in both eyes (fig $1 \mathrm{E}$ ).

The T2 weighted fast spin echo MRI of patients in the acute stage of LHON was compared with those of patients with unilateral acute optic neuritis. A high intensity signal area was seen in the involved retrobulbar optic nerve in five of the seven patients with optic neuritis (fig 2) and in the intracanalicular lesion in the sixth. One patient with papillitis showed normal findings in the orbital portion of the optic nerve complex. The Gd-DTPA enhanced T1 weighted MRI with fat suppression showed the enhanced optic nerve sheath of the affected eye.

\section{Discussion}

The T2 weighted fast spin echo sequence with fat suppression improves the resolution of the image and the signal to noise ratio while short- ening the imaging time, and is useful in routine clinical diagnosis. ${ }^{1011}$ The improved resolution may be useful in detecting small lesions of the optic nerve in the orbital portion. ${ }^{6812}$ With a repetition time of $4000 \mathrm{~ms}$ or longer, T2 weighted MRI is highly sensitive to regions of demyelination, oedema, inflammation, or gliosis. These regions clearly appear as an increased intensity of the signal in the nerve and sheath. ${ }^{6912}$

Abnormalities of the optic nerve with an increase in signal were seen by Kermode et al in all eight patients with LHON in whom STIR sequence images ( 0.5 tesla $M R$ imager) were obtained three months to 16 years after onset of disease. The increase in signal was located in the middle to posterior intraorbital portions of the nerves. In the present study, when we rescanned in early or intermediate stages of atrophy, the increase in signal was located throughout the nerves, and signals were higher in the anterior than in the posterior portion of each nerve. Increased optic nerve signals on MRI may perhaps represent gliosis of the optic nerve in the chronic stage of LHON with optic atrophy. ${ }^{13}$ In the study of Kermode et al, ${ }^{7}$ a patient in an acute stage of LHON with involvement of only one eye showed a unilat- 
eral high signal in the optic nerve corresponding to the affected eye. That led the authors to speculate that the high signal in the optic nerve indicated the presence of oedema. However, three months after disease onset, this patient was classified clinically as being in the early atrophic stage. ${ }^{1}$

An MRI study of 15 patients in different clinical stages of LHON by Dotti et al using T1 weighted images ( 0.5 tesla $M R$ imager) showed minor or no characteristic abnormalities in the optic nerve signal. Increased signals were seen in four of the 15 patients (one patient in the acute stage, two in the presymptomatic stage, and one in the chronic stage). The meaning of hyperintense signals in $\mathrm{T} 1$ weighted images in the different clinical stages of LHON is unknown.

Borruat et $a l^{14}$ described a 63 year old patient with LHON who initially had visual loss in the left eye, followed in 11 months by involvement of the right eye. An MRI with STIR sequence disclosed the presence of high signals in the left eye with optic atrophy, but no abnormal signals were seen in the right eye in the acute stage. Riordan-Eva et $a l^{15}$ found that non-enhancing high signal changes did not usually occur until at least three months after the onset of disease. Gass et $a l^{12}$ recently reported a high signal in the optic nerves of two chronic cases of LHON on T2 weighted fast spin echo MRI

High resolution fast spin echo MRI in the present study showed no characteristic abnormalities of the optic nerve complex in the orbit during the acute stage of LHON. Patients with optic neuritis, however, exhibited very high signals in the optic nerve. Enhancement of the optic nerve with Gd-DTPA enhanced T1 weighted MRI occurs when the blood-brain barrier is impaired by acute neuritis. ${ }^{16}$ This enhancement did not occur in LHON at an acute stage in the present study. This difference suggests that no great change in MRI occurs in the retrobulbar optic nerve during the acute stage of LHON. The MRI of the optic nerve gives information about the fluid and cellular environment of the axons.

Ophthalmoscopic and fluorescein angiographic findings in the acute stage of LHON have suggested that the primary locus of the lesion may be intraocular rather than retrobulbar. ${ }^{12}$ The present results of our MRI studies support this hypothesis.

This work was supported by a Grand in Aid for Research Committee on Chorioretinal Degenerations and Optic Atrophy from the Ministry of Health and Welfare of Japan.

1 Nikoskelainen E, Hoyt WF, Nummelin K. Ophthalmoscopic findings in Leber's hereditary optic neuropathy. II. The fundus findings in the affected family members. Arch Ophthalmol 1983;101:1059-68.

2 Nikoskelainen E, Hoyt WF, Nummelin K, Schatz H. Fundus findings in Leber's hereditary optic neuroretinopathy. III. Fluorescein angiographic studies. Arch Ophthalmol 1984;102:981-9.

3 Miller DH, Newton MR, van der Poel JC, et al. Magnetic resonance imaging of the optic nerve in optic neuritis. Neurology 1988;38:175-9.

4 Lee DH, Simon JH, Szumowski J, et al. Optic neuritis and orbital lesions: lipid-suppressed chemical shift MR imaging. Radiology 1991;179:543-6.

5 Guy J, Mao J, Bidgood Jr WD, Mancuso A, Quisling RG. Enhancement and demyelination of the intraorbital optic nerve. Fat suppression magnetic resonance imaging. Ophthalmology 1992;99:713-9.

6 Gass A, Moseley IF, Barker GJ, et al. Lesion discrimination in optic neuritis using high-resolution fat-suppressed fast spin-echo MRI. Neuroradiology 1996;38:317-21.

7 Kermode AG, Moseley IF, Kendall BE, et al. Magnetic resonance imaging in Leber's optic neuropathy. $f$ Neurol Neurosurg Psychiatry 1989;52:671-4.

8 Dotti MT, Caputo N, Signorini E, Federico A. Magnetic resonance imaging findings in Leber's hereditary optic neuropathy. Eur Neurol 1992;32:17-9.

9 Mashima Y, Oshitari K, Imamura Y, et al. High-resolution magnetic resonance imaging of the intraorbital optic nerve and subarachnoid space in patients with papilledema and optic atrophy. Arch Ophthalmol 1996;114:1197-203.

10 Mukherji SK, Tart RP, Fitzsimmons J, et al. Fat-suppressed MR of the orbit and cavernous sinus: comparison of fast MR of the orbit and cavernous sinus: comparison of fast
spin-echo and conventional spin-echo. AfNR Am $\mathcal{7}$ Neuroradiol 1994;15:1707-14.

11 Fulbright R, Panush D, Sze G, et al. MR of the head and neck: comparison of fast spin-echo and conventional spinecho sequences. AfNR Am F Neuroradiol 1994;15:767-73.

12 Gass A, Barker GJ, MacManus D, et al. High resolution magnetic resonance imaging of the anterior visual pathway in patients with optic neuropathies using fast spin echo and phased array local coils. F Neurol Neurosurg Psychiatry 1995;58:562-9.

13 Barnes D, McDonald WI, Landon DN, Johnson G. The characterization of experimental gliosis by quantitative nuclear magnetic resonance imaging. Brain 1988;111:8394.

14 Borruat F-X, Green WT, Graham EM, et al. Late onset Leber's optic neuropathy. Br f Ophthalmol 1992;76:571-3.

5 Riordan-Eva P, Sanders MD, Govan GG, et al. The clinical features of Leber's hereditary optic neuropathy defined by the presence of a pathogenic mitochondrial DNA mutation. Brain 1995;118:319-37.

16 Guy J, Mancuso A, Quisling RG, Beck R, Moster M. Gadolinium-DTPA-enhanced magnetic resonance imaging in optic neuropathies. Ophthalmology 1990;97:592600 . 